\title{
Significant Benefit of Multimodal Imaging: PET/CT Compared with PET Alone in Staging and Follow-up of Patients with Ewing Tumors
}

\author{
Hans U. Gerth ${ }^{1}$, Kai U. Juergens ${ }^{2}$, Uta Dirksen ${ }^{3}$, Joachim Gerss ${ }^{4}$, Otmar Schober ${ }^{1}$, and Christiane Franzius ${ }^{1}$ \\ ${ }^{I}$ Department of Nuclear Medicine, University Hospital Münster, Münster, Germany; ${ }^{2}$ Department of Clinical Radiology, University \\ Hospital Münster, Münster, Germany; ${ }^{3}$ Department of Pediatric Hematology and Oncology, University Hospital Münster, Münster, \\ Germany; and ${ }^{4}$ Department of Medical Informatics and Biomathematics, University Hospital Münster, Münster, Germany
}

Hybrid PET/CT was compared with PET alone in the staging and restaging of patients with Ewing tumor to assess the benefit of the combined imaging technique. Methods: A total of 163 ${ }^{18} \mathrm{~F}-\mathrm{FDG} \mathrm{PET} / \mathrm{CT}$ studies performed in 53 patients (age: range, 4-38 y; median, 16.5 y) with histopathologically confirmed Ewing tumor were evaluated retrospectively. All PET/CT studies included low-dose CT for attenuation correction; in 91 examinations, additional diagnostic chest CT was performed. PET and CT data were assessed independently by 2 nuclear medicine physicians and 2 radiologists, respectively. Finally, both datasets were fused by use of software and analyzed by all 4 reviewers (consensus reading). Each lesion was scored with a 5-point scale. Biopsy, imaging, or clinical follow-up served as a standard of reference. Receiver operating characteristic (ROC) analyses were performed to evaluate PET and PET/CT performance characteristics. To measure the abilities to detect and correctly localize tumor foci, localization ROC (L-ROC) curves were generated for PET. Results: A total of 609 lesions were detected by PET alone. The hybrid PET/CT technique resulted in a change of score in 160 of these lesions (26\%): higher scores in 23 lesions (4\%) and lower scores in 137 lesions (23\%). In 49 lesions detected by PET (8\%), the localization had to be changed after image fusion. Additionally, 124 (21\%) more lesions were found by PET/CT than by PET alone, resulting in a total of 733 lesions. As determined by lesion-based analysis, the sensitivity, specificity, and accuracy of PET were $71 \%, 95 \%$, and $88 \%$, respectively; the corresponding values for the hybrid PET/CT technique were $87 \%, 97 \%$, and $94 \%(P<0.0001)$. The areas under the curve in the ROC analysis were 0.82 for PET and 0.92 for $\mathrm{PET} / \mathrm{CT}(P<0.0001)$, and that in the L-ROC analysis was 0.66 for PET. Conclusion: PET/CT is significantly more accurate than PET alone for the detection and localization of lesions and improves staging for patients with Ewing tumor. The hybrid technique is superior to PET alone in terms of sensitivity, specificity, and accuracy, mainly because of the detection of new lesions.

\footnotetext{
Received Jul. 23, 2007; revision accepted Sep. 6, 2007.

For correspondence or reprints contact: Hans U. Gerth, PhD, Department of Nuclear Medicine, University Hospital Münster, Albert-Schweitzer-Strasse 33, 48149 Münster, Germany.

E-mail: huvgerth@uni-muenster.de

COPYRIGHT @ 2007 by the Society of Nuclear Medicine, Inc.
}

Key Words: PET; PET/CT; Ewing tumor; sarcoma; ROC analysis J Nucl Med 2007; 48:1932-1939

DOI: 10.2967/jnumed.107.045286

$\mathrm{A}_{\text {lthough the implementation of multimodal treatment }}$ concepts, including chemotherapy, surgery, and radiation therapy, has significantly improved the prognosis for patients with localized Ewing tumor, the prognosis for patients with primary disseminated disease is still poor, and optimized disease management is missing (1-3). The rate of survival of children with this second most common malignant childhood and adolescence bone tumor is about $60 \%$ for localized disease, and it is even worse for metastases $(1,4,5)$. The 5-y survival rate is less than $40 \%$ for patients with lung metastases and less than $20 \%$ for patients with osseous metastases and bone marrow infiltration $(6,7)$. Therefore, detection of metastases and exact disease staging are essential for the best possible treatment and outcome.

Detection and staging of cancer with noninvasive imaging modalities, such as CT or MRI, do not provide sufficient distinction between vital and necrotic tumor cells or exclude minimal malignant residues (8). Therefore, PET is increasingly being used as a powerful diagnostic method in the detection of glucose hypermetabolic malignancies (9). Additionally, PET allows monitoring of responses during therapy (10). However, the limited spatial resolution of PET hampers the precise anatomic localization of identified lesions. The combination of PET with the complementary diagnostic imaging modality $\mathrm{CT}$ in an integrated PET/CT scanner helps to overcome this limitation, and PET/CT increasingly is gaining acceptance in clinical settings $(11,12)$.

Studies concerning the diagnosis of malignant tumors in adult patients have shown that integrated PET/CT scanners are superior in terms of overall sensitivity and specificity to PET or CT alone $(11,13,14)$. In contrast, only a few studies have addressed integrated PET/CT for pediatric or adolescent malignancies (15-17), and further research is still needed (18-21). 
The interpretation of PET images in pediatric oncology remains challenging because artifacts with increased tracer uptake occur regularly in the adenoids, tonsils, or "brown fat" and can easily mimic false-positive malignant disease or impair the differentiation between normal and malignant tissues (22); the small size of children may further complicate image interpretation. Therefore, the morphologic information that is obtained by nondiagnostic (low-dose) CT performed for attenuation correction in modern hybrid PET/CT systems is of particular diagnostic value (18). ${ }^{18} \mathrm{~F}-$ FDG PET has been shown to detect osseous Ewing tumor lesions with a high sensitivity and a high specificity (2325). Furthermore, it has been proposed that ${ }^{18} \mathrm{~F}-\mathrm{FDG}$ uptake by Ewing tumors may have prognostic value and may predict a patient's clinical outcome as well as indicate the initial disease stage $(26,27)$. However, the value of integrated PET/CT for Ewing tumors has not been systematically evaluated to date.

The aim of this study was to evaluate the diagnostic accuracy and incremental value of PET/CT in comparison with PET for Ewing tumor.

\section{MATERIALS AND METHODS}

\section{Patient Population}

All patients included in this retrospective study (Table 1) fulfilled the following criteria: presence of histopathologically confirmed Ewing tumor; inclusion in EURO-E.W.I.N.G. 99 (EUROpean Ewing tumour Working Initiative of National Groups-Ewing Tumour Studies 1999), which was approved by the local ethics board; PET/CT examinations between January 2004 and June 2006; and follow-up of at least 6 mo.

All patients or their parents had given their written informed consent for inclusion in the EURO-E.W.I.N.G. 99 study, analysis of their clinical data for research purposes, and PET/CT examinations.

\section{Data Acquisition}

All PET/CT studies were performed with a hybrid scanner (Biograph Sensation 16; Siemens Medical Solutions), which consists of a full-ring PET tomograph (lutetium oxyorthosilicate crystals, picoelectronics) and a 16-detector-row CT component.

TABLE 1

Patient Characteristics and Timing and Clinical Indications of PET/CT Examinations

\begin{tabular}{lc}
\hline Characteristic or examination purpose & Value \\
\hline No. of patients (men/women) & $53(36 / 17)$ \\
Age (y) at examination date, range/mean & $4-38 / 16.5$ \\
Total no. of examinations & 163 \\
Examination purpose (no. of examinations) & \\
Initial staging & 13 \\
Therapy monitoring after chemotherapy & 17 \\
After surgical intervention & 46 \\
Follow-up after completion of therapy & 68 \\
or restaging & 19 \\
During relapse therapy & $22,7,6,1$, \\
No. of patients with 1, 2, 3, 4, and & and 17 \\
>4 examinations &
\end{tabular}

Details concerning PET/CT in pediatric patients have been published elsewhere (18). All patients were studied after fasting for at least $5 \mathrm{~h}$. Blood glucose levels at the time of ${ }^{18} \mathrm{~F}-\mathrm{FDG}$ application were less than $120 \mathrm{mg} / \mathrm{dL}$. Body-weight-adapted activity of ${ }^{18} \mathrm{~F}-\mathrm{FDG}$ (4 MBq/kg of body weight) was injected intravenously $60 \mathrm{~min}$ before the acquisition. Patients were positioned head first and supine in the PET/CT scanner. An identical field of view was chosen from the base of the skull to the feet for the PET scan and the low-dose CT scan. First, a low-dose CT scan with acquisition parameters adjusted for pediatric patients (18) was acquired during mild expiration for attenuation correction and location of anatomic landmarks. If clinically indicated, for example, to detect pulmonary metastases at initial diagnosis or for follow-up studies after systemic therapy, patients underwent additional, diagnostic CT of the chest during full inspiration after the intravenous application of contrast medium and with weightand size-adjusted acquisition parameters (18). Acquisition of the CT scan(s) was followed by an automatic table movement to the PET position; emission data were acquired for $4 \mathrm{~min}$ in each bed position.

\section{Data Reconstruction}

Low-dose CT datasets were reconstructed in an overlapping manner at a $3-\mathrm{mm}$ slice thickness with a $1.5-\mathrm{mm}$ reconstruction increment by use of standard soft-tissue reconstruction kernel B30 and standard lung and bone reconstruction kernels B50 and B60; the field of view $\left(<350 \mathrm{~mm}^{2}\right)$ was individually adapted to the body contours. ${ }^{18}$ F-FDG PET data were reconstructed iteratively by use of the ordered-subset expectation maximization algorithm with data from the low-dose CT used for attenuation correction.

If a contrast-enhanced CT scan of the chest during full inspiration was obtained, those $\mathrm{CT}$ data were reconstructed at a 1-mm slice thickness with an $0.8-\mathrm{mm}$ reconstruction increment by use of standard soft-tissue (B30) and lung (B50) reconstruction kernels and a field of view covering the entire chest cross section.

All reconstructed ${ }^{18} \mathrm{~F}-\mathrm{FDG}$ PET and CT data were transferred to a Leonardo workstation (VA 70; Siemens Medical Solutions) for further assessment.

\section{Imaging Analysis}

All PET/CT studies included low-dose CT for attenuation correction of PET data; in 91 examinations, chest CT was performed for diagnostic purposes. Fully diagnostic CT was performed only for initial staging, restaging in cases of confirmed or highly likely relapse, and known pulmonary metastases in followup. PET and CT data each were interpreted independently by 2 experienced nuclear medicine physicians and 2 experienced radiologists, respectively. The evaluating physicians knew the clinical background but were unaware of the results of other imaging modalities. In a final consensus reading, PET and CT images were analyzed again side by side as well as after software-based image fusion by all 4 reviewers. In each reading, the locations of all abnormalities were recorded, and the lesions were scored with a 5 -point scale $(28,29)$ : 5 , definitely malignant; 4 , probably malignant; 3 , equivocal; 2 , probably benign; and 1 , definitely benign or physiologic tracer uptake. When individual patients had multiple organ metastases (e.g., pulmonary metastases), a maximum of 4 lesions per organ were included for analysis to avoid the bias of a few studies with a very large number of lesions. 


\section{Reference Methods}

The site of each lesion was regarded as malignant when the site met at least one of the following criteria: the site was confirmed by histopathology, the site was confirmed by other imaging modalities (MRI, diagnostic CT, or bone scintigraphy), the site exhibited progressive disease in the follow-up period, or the site was accompanied by multiple PET- and CT-positive sites that met any of the first 3 criteria.

On follow-up PET scans, an increase in ${ }^{18}$ F-FDG uptake in a lesion was considered to represent the progression of tumor activity. On follow-up CT scans, an increase in the size of a whole lesion or of the extraosseous part of a lesion or a progressive permeative lytic lesion with a periosteal reaction was considered to be a sign of malignancy.

\section{Statistical Analysis and Data Interpretation}

Statistical analysis was performed on a lesion basis as well as on an examination basis. For examination-based analysis, lesionbased data were aggregated by examination, assigning the worst evaluation among all lesions within an examination, respectively (i.e., the highest single score of all identified lesions within an examination was assigned to the score of the whole examination). The results of PET and PET/CT were compared by use of a $5 \times 5$ contingency table. Differences in diagnostic performance were assessed with a marginal homogeneity test.

Sensitivity, specificity, accuracy, positive predictive value (PPV), and negative predictive value (NPV) were calculated in 3 different ways. In the first method, equivocal cases were not considered (scores 1 and 2: benign; scores 4 and 5: malignant). This method represents common clinical practice. Usually, equivocal foci were reevaluated with other modalities or observed with very close follow-up examinations. In the second method, equivocal foci were included and considered to be benign foci (scores 1-3: benign; scores 4 and 5: malignant). In the third method, equivocal foci were included and considered to be malignant foci (scores 1 and 2: benign; scores 3-5: malignant).

The ordinal data obtained with the 5-point scale were analyzed to create receiver operating characteristic (ROC) curves (30). To compare the areas under the curve (AUC) for PET and PET/CT, a nonparametric approach was applied (31). In conventional ROC analysis, the localization of detected lesions is ignored. Consequently, lesions are classified as true positive, even if the correct localization of a lesion has failed. In an alternative approach, the true-positive classification of a lesion requires not only its detection but also its correct localization (L-ROC analysis) (32). Therefore, L-ROC and ROC analyses were performed for the PET datasets. In addition, ROC analyses of different lesion subgroups, such as lesions located in lung tissue, soft tissue, and bones, were carried out.

$P$ values of $\leq 0.01$ were designated as statistically significant.

\section{RESULTS}

PET alone revealed a total of 609 lesions. A total of 124 additional sites $(21 \%)$ were detected by CT (low-dose CT or diagnostic chest CT). The total number of lesions identified by PET/CT therefore was 733. In addition, CT information resulted in changes in the scoring of foci (Table 2). Compared with PET alone, PET/CT resulted in higher scores for 23 foci (4\%) and lower scores for 137 foci $(23 \%)$ (supplemental Table 1; supplemental materials are available online only at http://jnm.snmjournals.org).

The frequency of equivocal scans was not reduced by PET/CT relative to PET (both 12\%). However, when additional foci detected by CT were excluded, the improved localization of PET/CT reduced the number of equivocal foci detected by PET from $76(12 \%)$ to $46(8 \%)$. The frequency of definitely benign lesions was increased from $227(37 \%)$ to $329(54 \%)$ by PET/CT. Furthermore, in $8 \%$ ( $n=49$ ) of the 609 foci detected by PET, the additional information and exact image fusion resulted in changes in localization (Table 3). When only lesions with changes in localization were considered, the number of equivocal lesions was reduced from 14\% (PET alone) to 4\% (PET/ $\mathrm{CT}$ ), and the number of definitely benign lesions was increased from 6\% (PET alone) to $27 \%$ (PET/CT). Of the 124 foci that were detected only by CT, $65 \%(n=81)$ were

TABLE 2

Results of PET and PET/CT Examinations and Changes After Image Fusion

\begin{tabular}{lc}
\hline \multicolumn{1}{c}{ Result } & No. (\%) of lesions \\
\hline Foci detected by PET alone & 609 \\
Additional foci detected by CT & 124 \\
Foci detected by PET/CT & 733 \\
Changes in equivocal foci detected by PET (PET score, 3) after image fusion & $76(100)$ \\
PET/CT score & $15(20)$ \\
1 (definitely benign or physiologic) & $18(24)$ \\
2 (probably benign) & $36(47)$ \\
3 (equivocal) & $3(4)$ \\
$\quad$ (probably malignant) & $4(5)$ \\
5 (definitely malignant) & $137(23)$ \\
Changes after image fusion: lesion-based analysis $(n=609)$ & $23(4)$ \\
Lower scores for foci detected by PET & $49(8)$ \\
Higher scores for foci detected by PET & $37(23)$ \\
Change in localization of foci detected by PET & $19(12)$ \\
Changes after image fusion: examination-based analysis $(n=163)$ & \\
Lower scores & \\
Higher scores &
\end{tabular}


TABLE 3

Localization of Foci

\begin{tabular}{lccc}
\hline & \multicolumn{3}{c}{ No. (\%) } \\
\cline { 2 - 4 } Localization & $\begin{array}{c}\text { Foci } \\
\text { detected } \\
\text { by PET/CT }\end{array}$ & $\begin{array}{c}\text { New foci } \\
\text { detected } \\
\text { by CT }\end{array}$ & $\begin{array}{c}\text { Final localization of } \\
\text { foci with changes } \\
\text { in localization }\end{array}$ \\
\hline Lungs & $106(15)$ & $81(65)$ & $5(10)$ \\
Skeleton & $275(38)$ & $27(22)$ & $22(45)$ \\
Soft tissue & $201(27)$ & $16(13)$ & $17(35)$ \\
Physiologic & $151(21)$ & $0(0)$ & $5(10)$ \\
Total & $733(100)$ & $124(100)$ & $49(100)$ \\
\hline
\end{tabular}

located in the lungs (Fig. 1). Of the 81 pulmonary foci, 61 were detected by full diagnostic chest $\mathrm{CT}$ and the remaining 20 were detected by low-dose CT (supplemental Table 2).

The standard of reference was histology for 72 lesions, other imaging methods for 84 lesions, and clinical and imaging follow-up for 577 lesions. The results of scoring on lesion and examination bases and the classification parameters are shown in Table 4. All parameters could be increased by consideration of the information from CT, but sensitivity showed the greatest change (supplemental Table 3).

The differences in the AUCs for PET and PET/CT in the lesion-based ROC analyses including (Fig. 2A) and excluding (Fig. 2B) new foci detected by CT were significant. On an examination basis, the AUC was enhanced when CT information was considered, but the result did not reach the level of significance (Fig. 2C). ROC analyses of the different localization subgroups showed improvement in all localizations by PET/CT in comparison with PET alone (Fig. 3A-3C). The greatest improvement was achieved for pulmonary foci (AUC for PET, 0.66; AUC for PET/CT, $0.89 ; P<0.0001)$.

\section{DISCUSSION}

To our knowledge, this report is the first describing the diagnostic impact of integrated PET/CT in comparison with PET for patients with Ewing tumor. The results of the present study demonstrate that the combined technique exhibits superior performance. This result is mainly attributable to the detection of additional lesions and the improvement of lesion localization by CT examination.

\section{Additional Foci Detected by CT}

To increase the sensitivity of imaging modalities, it is necessary to reduce the number of false-negative results. Several lesions are seen on low-dose CT but not on PET (e.g., lung lesions) and vice versa (e.g., osseous lesions) (33). A total of 124 new foci detected by CT (20\%) were not seen by PET alone; $65 \%$ of the new foci detected by CT were located in the lungs. These findings are in concordance with those of earlier PET studies in patients with Ewing tumor, which showed a low sensitivity of PET for the detection of pulmonary metastases $(24,25,27)$. Although none of the new foci detected by CT were of physiologic origin, $55 \%$ were scored as probably or definitely benign. A large portion, however, were scored as equivocal (36\%), and only $19 \%$ were scored as probably or definitely malignant. However, among the 124 new lesions detected by CT, 23 additional true-positive foci were not detected by PET alone (an additional 12\% truepositive malignant foci among the total of 193 malignant lesions). These results are relevant for staging and further therapeutic management.

\section{Mislocalization}

Mislocalization is one of the major problems of interpretation of images obtained by PET alone. Typical examples are intrapulmonary metastases falsely localized by PET interpretation in adjacent structures, such as the pleura, ribs, or mediastinum (34). The additional anatomic information provided by the CT component allows mislocalization to be avoided. Especially in pediatric patients, anatomic correlation is very helpful because of the smaller anatomy and the different organ proportions of pediatric patients relative to adult patients.

To quantify the diagnostic performance of PET, including correct localization, L-ROC curves were generated. Simultaneously detecting and properly localizing lesions reduced the AUCs of the L-ROC curves to 0.66 in the lesion-based analysis and to 0.78 when additional foci detected by $\mathrm{CT}$ were excluded. The corresponding values
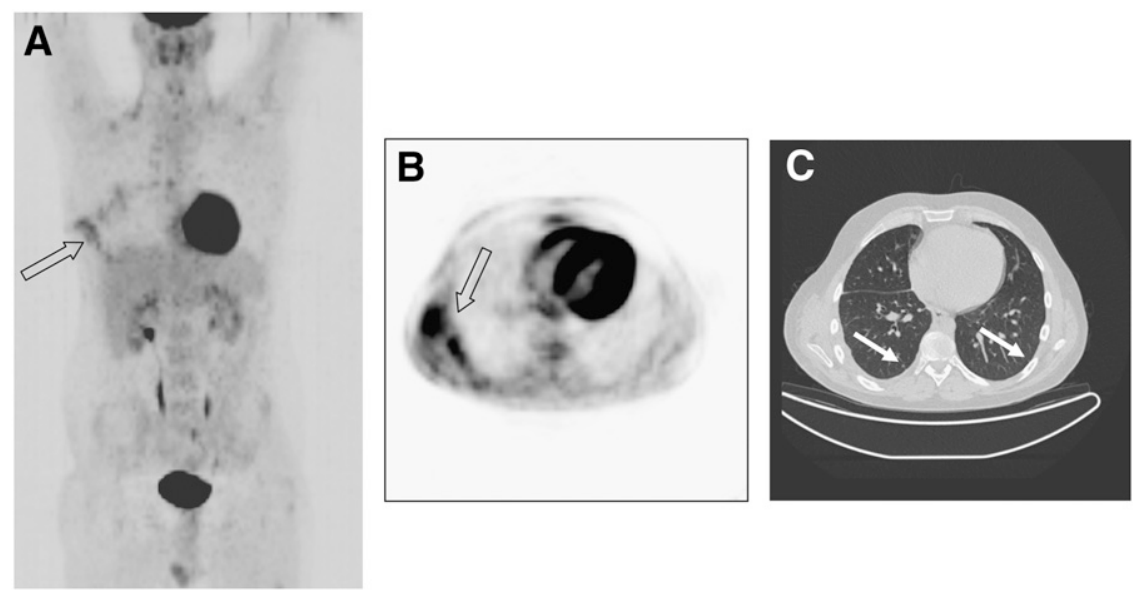

FIGURE 1. Images for 23-y-old male patient who had Ewing sarcoma in right tibia years ago and pulmonary relapse, with resection of pulmonary metastases 1 mo earlier. (A and B) ${ }^{18} \mathrm{~F}-\mathrm{FDG}$ PET demonstrates postoperative increased glucose metabolism in right lateral thorax (open arrow) but no intrapulmonary hypermetabolic foci. (C) Thoracic CT in so-called pulmonary window shows postoperative scar and multiple new small pulmonary lesions (arrows), which increased in size on follow-up and were classified as malignant. (A) ${ }^{18} \mathrm{~F}$-FDG PET maximum-intensity projection. (B) Transverse slice from ${ }^{18} \mathrm{~F}-$ FDG PET. (C) Thoracic CT. 
TABLE 4

Lesion- and Examination-Based Sensitivity, Specificity, Accuracy, PPV, and NPV of PET and PET/CT Examinations

\begin{tabular}{|c|c|c|c|c|c|c|c|}
\hline \multirow[b]{3}{*}{ Status of equivocal foci } & \multirow[b]{3}{*}{ Parameter } & \multicolumn{6}{|c|}{ Value of parameter, \% (95\% exact binomial confidence interval) } \\
\hline & & \multicolumn{2}{|c|}{$\begin{array}{l}\text { Lesion-based } \\
\text { analysis }\end{array}$} & \multicolumn{2}{|c|}{$\begin{array}{l}\text { Lesion-based analysis } \\
\text { with lesions detected } \\
\text { by CT only excluded }\end{array}$} & \multicolumn{2}{|c|}{$\begin{array}{c}\text { Examination-based } \\
\text { analysis }\end{array}$} \\
\hline & & $\begin{array}{c}\text { PET } \\
\left(n=640^{\star}\right)\end{array}$ & $\begin{array}{c}\mathrm{PET} / \mathrm{CT} \\
(n=733)\end{array}$ & $\begin{array}{c}\text { PET } \\
(n=609)\end{array}$ & $\begin{array}{c}\mathrm{PET} / \mathrm{CT} \\
(n=609)\end{array}$ & $\begin{array}{c}\text { PET } \\
(n=163)\end{array}$ & $\begin{array}{l}\text { PET/CT } \\
(n=163)\end{array}$ \\
\hline \multirow[t]{5}{*}{ Excluded } & Sensitivity & $71(64-78)$ & $87(81-91)$ & $87(80-92)$ & $86(79-91)$ & $92(81-98)$ & $96(87-100)$ \\
\hline & Specificity & $95(93-97)$ & 97 (95-99) & $95(93-97)$ & 97 (95-99) & $88(79-94)$ & $92(84-97)$ \\
\hline & Accuracy & $88(85-91)$ & $94(93-97)$ & $93(93-97)$ & $94(93-97)$ & $90(86-98)$ & $94(91-100)$ \\
\hline & PPV & $87(80-92)$ & $92(87-96)$ & $87(80-92)$ & $91(85-95)$ & $84(72-93)$ & $89(78-96)$ \\
\hline & NPV & $88(85-91)$ & 95 (93-97) & 95 (93-97) & 95 (93-97) & $94(86-98)$ & $97(91-100)$ \\
\hline \multirow[t]{5}{*}{ Classified as benign } & Sensitivity & $63(56-70)$ & 77 (70-82) & 75 (68-82) & 77 (70-83) & $80(68-89)$ & $85(73-93)$ \\
\hline & Specificity & $96(94-98)$ & 98 (96-99) & 96 (94-98) & 97 (95-99) & $91(84-96)$ & $94(88-98)$ \\
\hline & Accuracy & $86(82-89)$ & $92(90-94)$ & $90(89-94)$ & 92 (89-94) & 87 (81-94) & $91(84-96)$ \\
\hline & PPV & 87 (80-92) & $92(87-96)$ & 87 (80-92) & 91 (85-95) & $84(72-93)$ & $89(78-96)$ \\
\hline & NPV & $86(82-89)$ & $92(90-94)$ & 91 (89-94) & 92 (89-94) & 89 (81-94) & $92(84-96)$ \\
\hline \multirow{5}{*}{ Classified as malignant } & Sensitivity & $74(67-80)$ & $88(83-92)$ & $88(82-93)$ & 87 (81-92) & $93(84-98)$ & $97(88-100)$ \\
\hline & Specificity & $84(80-87)$ & $85(82-88)$ & $84(80-87)$ & 91 (88-93) & $65(55-74)$ & $70(60-79)$ \\
\hline & Accuracy & $81(85-91)$ & $86(93-97)$ & $85(93-97)$ & 90 (93-97) & 75 (86-98) & $80(91-100)$ \\
\hline & PPV & $66(59-72)$ & $67(61-73)$ & $66(59-72)$ & 77 (70-83) & $61(50-71)$ & 65 (54-75) \\
\hline & NPV & $88(85-91)$ & $95(93-97)$ & $95(93-97)$ & 95 (93-97) & $94(86-98)$ & $97(91-100)$ \\
\hline
\end{tabular}

*Including 31 malignant foci detected by CT but not by PET alone and therefore graded as benign (score, 1).

for PET/CT were 0.92 and 0.92 and underlined the outstanding diagnostic performance of the hybrid technique.

In $8 \%$ of all foci detected by PET, the localization had to be changed after the CT information was considered. The majority of mislocalized foci $(90 \%)$ were actually located in the skeleton, soft tissues, or physiologic foci. The question that arises is whether such changes in localization have an impact on patient staging and therapeutic management. In lesions with changes in localization, the precise anatomic information provided by CT helps to reduce the frequency of equivocal lesions (PET, 14\%; PET/CT, 4\%) and increases the frequency of probably benign or physiologic foci (PET, 6\%; PET/CT, 27\%). The frequency of malignant or probably malignant foci does not change when anatomic information is included. Furthermore, localization of metastases is a major prognostic factor in Ewing tumor. Therefore, therapeutic management for patients with metastases depends on the affected organs (lungs, pleura, bone, bone marrow, and other locations) (5-7). In particular, the prognosis in patients with osseous lesions is very poor $(5,7)$, and it is essential that these patients receive intensive therapy. The identification of all metastatic sites and the correct localization of lesions are important for staging and for making therapeutic management decisions, because metastasectomy or external radiation of all tumor foci provides a curative approach.

\section{Organ-Specific Analyses}

ROC analyses of different organ-specific subgroups showed that PET/CT was significantly more accurate than PET in all evaluated locations and that pulmonary foci displayed the greatest increase in AUCs (PET, 0.66; PET/ CT, 0.89; $P<0.0001)$. These findings are in accordance with those of former studies showing that the sensitivity of PET alone is low and inferior to that of spiral CT in detecting pulmonary metastases and may be attributable to respiration movements during emission acquisition $(23,24)$. The PET/CT technique offers both the high sensitivity of PET in the detection of osseous and soft-tissue lesions $(23,25)$ and the high sensitivity of CT in the detection of lung metastases within a single examination.

A comparison of low-dose CT and full diagnostic CT of the chest with regard to the detection of pulmonary metastases was not addressed in the present study. The question as to whether the sensitivity of low-dose CT is high enough to exclude pulmonary metastases remains and should be evaluated in further studies.

\section{Radiation Exposure}

Although PET plays an important role in the diagnostic process for patients with Ewing tumor, radiation exposure during an examination often is of great concern $(18,19)$. For pediatric patients, special weight-adapted protocols are used, with an effective doses of about $5 \mathrm{mSv}$ in small children and in adolescents $(35,36)$. The effective dose of low-dose CT scans is reduced to as little as $1 / 26$ that of diagnostic CT scans in adults, which is about $0.7 \mathrm{mSv}$ for whole-body low-dose CT (37). However, the results of the present study and our clinical experience show that the improved diagnostic results obtained with hybrid PET/CT with low-dose CT are more valuable for making therapeutic 

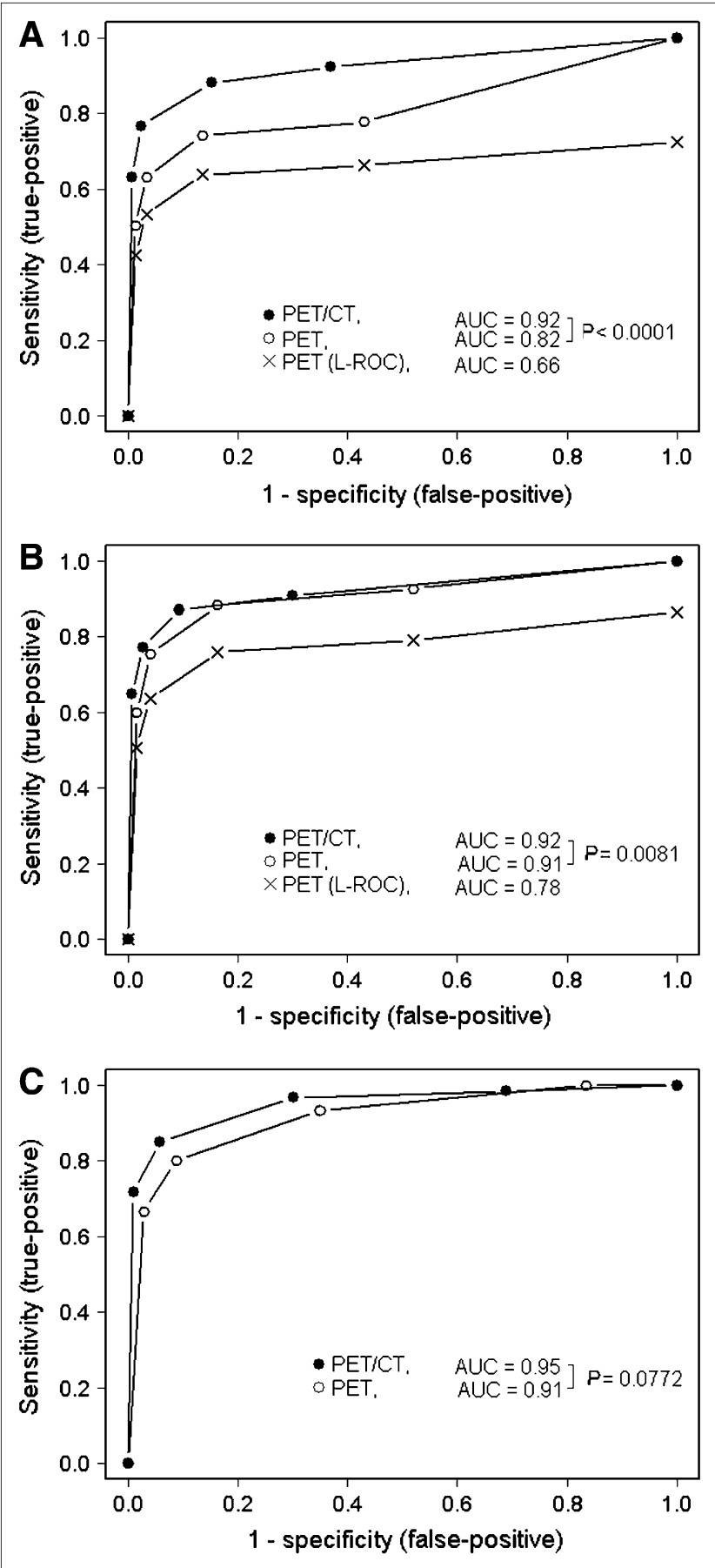

FIGURE 2. (A) Lesion-based ROC analysis ( $n=733$ ). (B) Lesion-based ROC analysis with additional foci detected by CT excluded ( $n=609)$. (C) Examination-based ROC analysis $(n=$ 163). Additional L-ROC curves classified lesion as true positive only when determination of whether lesion was malignant or benign and localization were correct.

management decisions and outweigh the risk that additional radiation exposure may harm the patient.

\section{Limitations}

Only a small number of PET and CT findings could be compared with histopathologic findings, possibly biasing
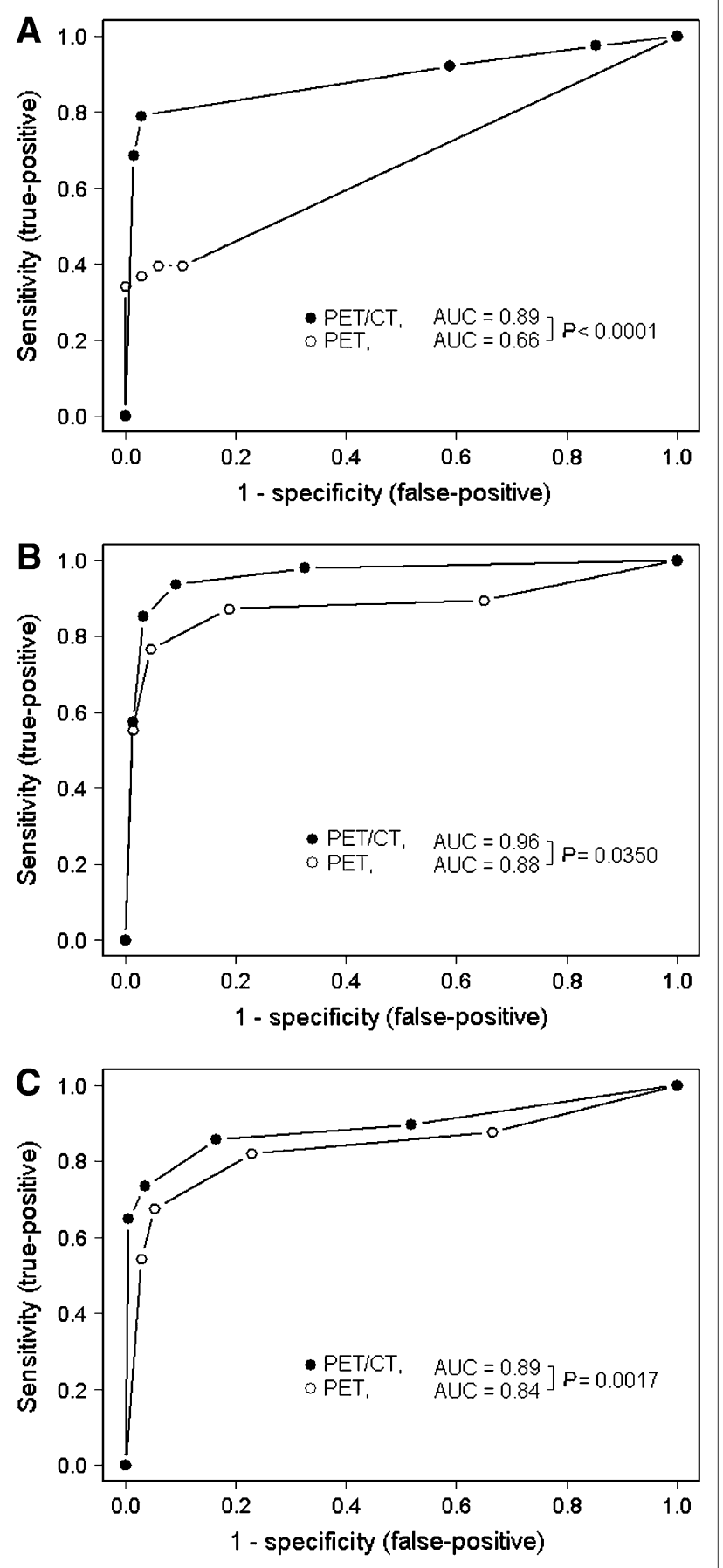

FIGURE 3. ROC analysis of pulmonary foci $(n=106)$ (A), soft tissue $(n=201)(B)$, and skeletal foci $(n=275)(C)$.

the specificity of the results. This lack of a histologic gold standard is a common problem in imaging studies and in clinical situations. Therefore, the status "positive for malignancy" was based on a clinically accepted concept $(11,13,14)$. A falsely high sensitivity of both PET and PET/CT could be attributable to the fact that metastases that were not revealed by any imaging modality were not considered. To minimize potential biases, a follow-up of at least 6 mo was adopted to exclude metastatic disease. 
Furthermore, other routine staging methods, that is, MRI of the primary tumor site and bone scintigraphy, were considered. The aim of the present study was to evaluate whether PET and PET/CT were able to depict viable tumor tissue and to provide correct information for staging and restaging. A systematic comparison with MRI, especially whole-body MRI, has not yet been performed.

\section{CONCLUSION}

There are inherent advantages of PET/CT over PET in lesion detection, determination of malignancy, proper localization, and therefore overall staging for patients with Ewing tumor. In agreement with the literature, the low sensitivity of PET for the depiction of small lung metastases could be enhanced by the CT component. Better lesion localization improves the determination of malignant lesions, resulting in fewer equivocal lesions. The beneficial properties of PET/CT are reflected in the superior sensitivity, specificity, accuracy, PPV, and NPV of this technique compared with PET alone. Image fusion reduces falsepositive and false-negative results in lesion-based analysis as well as examination-based analysis. This fact has direct consequences for individual therapeutic management decisions, because more precise staging reduces the number of patients who are overtreated or undertreated.

Hybrid PET/CT examination is recommended for staging and restaging in patients with Ewing tumor.

\section{ACKNOWLEDGMENTS}

The authors thank Marilyn Law, $\mathrm{PhD}$, for reviewing the article with respect to the English language. The authors also gratefully acknowledge the assistance of the technologists of the Department of Nuclear Medicine and the Department of Clinical Radiology in performing the PET/ CT scans. Many thanks are also due to all team members of the Department of Nuclear Medicine, the Department of Clinical Radiology, and the Ewing Sarcoma Study Group for their kind efforts, especially Walter Heindel, Thomas Allkemper, Rainald Bachmann, Stefan C. Krämer, Harald Seifarth, Johannes Wessling, and Dag Wormanns of the Department of Clinical Radiology; Jan B. Bremer, Klaudia Hermann, Markus Löffler, Michaela Pixberg, Burkhard Riemann, and Matthias Weckesser of the Department of Nuclear Medicine; and Heribert Jürgens, Gabriele BraunMunzinger, and Regina Kloss of the Ewing Sarcoma Study Group.

\section{REFERENCES}

1. Paulussen M, Ahrens S, Dunst J, et al. Localized Ewing tumor of bone: final results of the cooperative Ewing's Sarcoma Study CESS 86. J Clin Oncol. 2001;19:1818-1829.

2. Barker LM, Pendergrass TW, Sanders JE, et al. Survival after recurrence of Ewing's sarcoma family of tumors. J Clin Oncol. 2005;23:4354-4362.

3. Gerth HU, Rompel A, Krebs B, et al. Cytotoxic effects of novel polyoxotungstates and a platinum compound on human cancer cell lines. Anticancer Drugs. 2005;16:101-106.
4. Grier HE, Krailo MD, Tarbell NJ, et al. Addition of ifosfamide and etoposide to standard chemotherapy for Ewing's sarcoma and primitive neuroectodermal tumor of bone. N Engl J Med. 2003;20:694-701.

5. Cotterill SJ, Ahrens S, Paulussen M, et al. Prognostic factors in Ewing's tumor of bone: analysis of 975 patients from the European Intergroup Cooperative Ewing's Sarcoma Study Group. J Clin Oncol. 2000;18:3108-3114.

6. Paulussen M, Ahrens S, Craft AW, et al. Ewing's tumors with primary lung metastases: survival analysis of 114 (European Intergroup) Cooperative Ewing's Sarcoma Studies patients. J Clin Oncol. 1998;16:3044-3052.

7. Paulussen M, Ahrens S, Burdach S, et al. Primary metastatic (stage IV) Ewing tumor: survival analysis of 171 patients from the EICESS studies. European Intergroup Cooperative Ewing Sarcoma Studies. Ann Oncol. 1998; 9:275-281.

8. MacVicar AD, Olliff JF, Pringle J, et al. Ewing sarcoma: MR imaging of chemotherapy-induced changes with histologic correlation. Radiology. 1992; 184:859-864.

9. Rohren EM, Turkington TG, Coleman RE. Clinical applications of PET in oncology. Radiology. 2004;231:305-332.

10. Hawkins DS, Rajendran JG, Conrad EU III, Bruckner JD, Eary JF. Evaluation of chemotherapy response in pediatric bone sarcomas by [F-18]-fluorodeoxy-Dglucose positron emission tomography [published erratum appears in Cancer. 2003;97:3130]. Cancer 2002;94:3277-3284.

11. von Schulthess GK, Steinert HC, Hany TF. Integrated PET/CT: current applications and future directions. Radiology. 2006;238:405-422.

12. Ell PJ. The contribution of PET/CT to improved patient management. $\mathrm{Br} J$ Radiol. 2006;79:32-36.

13. Coleman RE, Delbeke D, Guiberteau MJ, et al. Concurrent PET/CT with an integrated imaging system: intersociety dialogue from the joint working group of the American College of Radiology, the Society of Nuclear Medicine, and the Society of Computed Body Tomography and Magnetic Resonance. J Nucl Med. 2005;46:1225-1239.

14. Wechalekar K, Sharma B, Cook G. PET/CT in oncology: a major advance. Clin Radiol. 2005;60:1143-1155.

15. Franzius C, Hermann K, Weckesser M, et al. Whole-body PET/CT with ${ }^{11} \mathrm{C}$ meta-hydroxyephedrine in tumors of the sympathetic nervous system: feasibility study and comparison with ${ }^{123}$ I-MIBG SPECT/CT. J Nucl Med. 2006;47:16351642 .

16. Rhodes MM, Delbeke D, Whitlock JA, et al. Utility of FDG-PET/CT in followup of children treated for Hodgkin and non-Hodgkin lymphoma. J Pediatr Hematol Oncol. 2006;28:300-306.

17. Miller E, Metser U, Avrahami G, et al. Role of ${ }^{18} \mathrm{~F}-\mathrm{FDG}$ PET/CT in staging and follow-up of lymphoma in pediatric and young adult patients. J Comput Assist Tomogr. 2006;30:689-694.

18. Franzius C, Juergens KU, Schober O. Is PET/CT necessary in paediatric oncology? For. Eur J Nucl Med Mol Imaging. 2006;33:960-965.

19. Hahn K, Pfluger T. Is PET/CT necessary in paediatric oncology? Against. Eur J Nucl Med Mol Imaging. 2006;33:966-968.

20. Kaste SC. Issues specific to implementing PET-CT for pediatric oncology: what we have learned along the way. Pediatr Radiol. 2004;34:205-213.

21. McCarville MB, Christie R, Daw NC, et al. PET/CT in the evaluation of childhood sarcomas. AJR. 2005;184:1293-1304.

22. Kaste SC, Howard SC, McCarville EB, et al. ${ }^{18}$ F-FDG-avid sites mimicking active disease in pediatric Hodgkin's. Pediatr Radiol. 2005;35:141-154.

23. Franzius C, Daldrup-Link HE, Wagner-Bohn A, et al. FDG-PET for detection of recurrences from malignant primary bone tumors: comparison with conventional imaging. Ann Oncol. 2002;13:157-160.

24. Franzius C, Daldrup-Link HE, Sciuk J, et al. FDG-PET for detection of pulmonary metastases from malignant primary bone tumors: comparison with spiral CT. Ann Oncol. 2001;12:479-486.

25. Franzius C, Sciuk J, Daldrup-Link HE, et al. FDG-PET for detection of osseous metastases from malignant primary bone tumours: comparison with bone scintigraphy. Eur J Nucl Med. 2000;27:1305-1311.

26. Gyorke T, Zajic T, Lange A, et al. Impact of FDG PET for staging of Ewing sarcomas and primitive neuroectodermal tumours. Nucl Med Commun. 2006;27: $17-24$.

27. Hawkins DS, Schuetze SM, Butrynski JE, et al. $\left[{ }^{18} \mathrm{~F}\right]$ Fluorodeoxyglucose positron emission tomography predicts outcome for Ewing sarcoma family of tumors. J Clin Oncol. 2005;23:8828-8834.

28. Kim JH, Czernin J, Allen-Auerbach MS, et al. Comparison between ${ }^{18}$ F-FDG $\mathrm{PET}$, in-line PET/CT, and software fusion for restaging of recurrent colorectal cancer. J Nucl Med. 2005;46:587-595.

29. Even-Sapir E, Parag Y, Lerman H, et al. Detection of recurrence in patients with rectal cancer: PET/CT after abdominoperineal or anterior resection. Radiology. 2004;232:815-822. 
30. Gönen M, Panageas KS, Larson SM. Statistical issues in analysis of diagnostic imaging experiments with multiple observations per patient. Radiology. 2001;221:763-767.

31. DeLong ER, DeLong DM, Clarke-Pearson DL. Comparing the areas under two or more correlated receiver operating characteristic curves: a nonparametric approach. Biometrics. 1988;44:837-845.

32. Starr SJ, Metz CE, Lusted LB, et al. Visual detection and localization of radiographic images. Radiology. 1975;116:533-538.

33. Delbeke D, Coleman RE, Guiberteau MJ, et al. Procedure guideline for tumor imaging with ${ }^{18} \mathrm{~F}$-FDG PET/CT 1.0 [published erratum appears in $\mathrm{J} \mathrm{Nucl} \mathrm{Med}$. 2006:47:903]. J Nucl Med. 2006;47:885-895.
34. Romer W, Nomayr A, Greess H, et al. Retrospective interactive rigid fusion of (18)F-FDG-PET and CT: additional diagnostic information in melanoma patients. Nuklearmedizin. 2006;45:88-95.

35. Lassmann M, Biassoni L, Monsieurs M, et al. The new EANM paediatric dosage card. Eur J Nucl Med Mol Imaging. 2007;34:796-798.

36. International Commission on Radiological Protection. ICRP publication 80: radiation dose to patients from radiopharmaceuticals. In: Annals of the ICRP. Vol 28/3. New York, NY: Elsevier; 2000.

37. Wu TH, Chu TC, Huang YH, et al. A positron emission tomography/computed tomography (PET/CT) acquisition protocol for CT radiation dose optimization. Nucl Med Commun. 2005;26:323-330. 